\title{
A Statistical Cohomogeneity One Metric on the Upper Plane with Constant Negative Curvature
}

\author{
Limei Cao, ${ }^{1}$ Didong Li, ${ }^{2}$ Erchuan Zhang, ${ }^{2}$ Zhenning Zhang, ${ }^{3}$ and Huafei Sun ${ }^{2}$ \\ ${ }^{1}$ School of Mathematics and Physics, University of Science and Technology Beijing, Beijing 100083, China \\ ${ }^{2}$ School of Mathematics and Statistics, Beijing Institute of Technology, Beijing 100081, China \\ ${ }^{3}$ College of Applied Sciences, Beijing University of Technology, Beijing 100124, China \\ Correspondence should be addressed to Huafei Sun; huafeisun@bit.edu.cn
}

Received 24 September 2014; Revised 15 December 2014; Accepted 15 December 2014; Published 30 December 2014

Academic Editor: Carlo Cattani

Copyright (C) 2014 Limei Cao et al. This is an open access article distributed under the Creative Commons Attribution License, which permits unrestricted use, distribution, and reproduction in any medium, provided the original work is properly cited.

we analyze the geometrical structures of statistical manifold $S$ consisting of all the wrapped Cauchy distributions. We prove that $S$ is a simply connected manifold with constant negative curvature $K=-2$. However, it is not isometric to the hyperbolic space because $S$ is noncomplete. In fact, $S$ is approved to be a cohomogeneity one manifold. Finally, we use several tricks to get the geodesics and explore the divergence performance of them by investigating the Jacobi vector field.

\section{Introduction}

So far, more and more geometric approaches have been applied to various fields such as in statistics, physics, and control [1-6], by analyzing their complexity characterizations or giving their solutions.

Entropic dynamics is a theoretical framework constructed on statistical manifolds to explore the possibility of laws of physics [7]. Until now, researchers have tried to study a variety of entropic dynamic models from the viewpoint of information geometry $[4-6,8]$. In [8], two entropic dynamical models are considered, in which the stability of the Jacobi vector fields on Riemannian manifolds is investigated. In [4], authors explored the geometric structures and instability of entropic dynamical models. Information geometric characterization of fractional Brownian motions was considered in [5]. Hamiltonian dynamics are studied from the viewpoint of geometry in [6].

In probability theory and directional statistics [9], a wrapped probability distribution is a continuous probability distribution, whose state can be considered as lying on a unit $n$-sphere $S^{n}$. Examples include the Bingham distribution [10], the Kent distribution (or the Fisher-Bingham distribution) [11], and the von Mises-Fisher distribution [9, 12]. Particularly for one-dimensional cases, a wrapped distribution consists of points on the unit circle. For instance, a wrapped Cauchy distribution $[9,13,14]$

$$
p(x ; \mu, \gamma)=\frac{1}{2 \pi} \frac{\sinh \gamma}{\cosh \gamma-\cos (x-\mu)}
$$

is a wrapped probability distribution that results from the "wrapping" of the Cauchy distribution around the unit circle, where apparently $x \in[-\pi, \pi)$ which is parameterized from the unit circle, $\gamma \in R$, and $\mu>0$. The functions sinh and cosh are, respectively, the hyperbolic sine function and the hyperbolic cosine function. As the random variable $x \in$ $[-\pi, \pi]$, the wrapped probability distribution can be considered as an angular probability to measure the directional probability. Therefore, it has been widely applied in the field of random walk for studying animal movements $[15,16]$, such as identifying fishing trip behavior and estimating fishing effort [17], and ringing recoveries of pied flycatchers [18].

Assume that all information relevant to dynamical evolution of the model can be obtained from the associated probability distribution, which is the wrapped Cauchy distribution in our consideration. We will call this model the WCED (wrapped Cauchy entropic dynamical) model for simplicity.

The remainder of the paper is organized as follows. In Section 2, the geometric structures, including the Fisher metric and the sectional curvature of the WCED, are calculated. 
In Section 3, this manifold is proved to be simply connected but not complete, hence not isometric to the hyperbolic space. However, it is proved to be a cohomogeneity one manifold by Theorem 14. In Section 4, several tricks are used based on some special properties to get geodesics and the Jacobi vector fields. The geodesics are the sets of fixed points of isometric transformations, which include symmetries to lines paralleled to $y$-axis. Meanwhile, the Jacobi vector fields are calculated. Then the instability of the geodesic spreads is analyzed via the behaviors of the the Jacobi vector field.

Remark 1. It is well known that the upper plane $\mathbb{R}_{+}^{2}$ admits a hyperbolic metric $g_{H}$ with constant negative curvature. That is,

$$
g_{H}=\left[\begin{array}{cc}
\frac{1}{y^{2}} & 0 \\
0 & \frac{1}{y^{2}}
\end{array}\right] \text {. }
$$

However, in this paper, we give another metric on $\mathbb{R}_{+}^{2}$ with constant negative curvature but not isometric to the hyperbolic space.

\section{Geometric Structures of the WCED Model}

From the theory of information geometry, one can define an $m$-dimensional statistical manifold $M$ over unit spheres, which is a set of probability densities; namely,

$$
M=\{p(x ; \theta)\} .
$$

The parameter $\theta=\left(\theta^{1}, \theta^{2}, \ldots, \theta^{m}\right)$ plays the role of coordinate systems. The Fisher information matrix $g$, acting as a Riemannian metric, is then given by the expectation

$$
\left(g_{i j}\right)=\left(E\left[\left(\partial_{i} l\right)\left(\partial_{j} l\right)\right]\right)
$$

where $l(x ; \theta):=\ln p(x ; \theta)$ and $\partial_{i}:=\partial / \partial \theta^{i}$. On a statistical manifold, the Riemannian connection coefficient $\Gamma_{i j k}$ is (see, e.g., $[1,2,19-21])$

$$
\Gamma_{i j k}=E\left[\left(\partial_{i} \partial_{j} l\right)\left(\partial_{k} l\right)\right]+\frac{1-\alpha}{2} E\left[\left(\partial_{i} l\right)\left(\partial_{j} l\right)\left(\partial_{k} l\right)\right],
$$

which can be expressed as a classical formula $\Gamma_{i j k}=$ $(1 / 2)\left(\partial_{i} g_{j k}+\partial_{j} g_{k i}-\partial_{k} g_{i j}\right)$ equivalently.

If $\left(\theta^{1}, \theta^{2}, \ldots, \theta^{m}\right)$ is a local coordinate of $M$, then for any vector field $X$ on $M$, one has $X=X^{i} \partial_{i}$, where $\left\{X^{i}\right\}$ are smooth functions with respect to $\left(\theta^{1}, \theta^{2}, \ldots, \theta^{m}\right)$. The Einstein summation convention is used here and all through the paper. With the Riemannian connection, the curvature tensor is defined by $[19,20]$

$$
R(X, Y) Z=\nabla_{X} \nabla_{Y} Z-\nabla_{Y} \nabla_{X} Z-\nabla_{[X, Y]} Z,
$$

and the Riemannian curvature tensor is

$$
R(X, Y, Z, W)=g(R(Z, W) X, Y),
$$

where $X, Y, Z, W$ are smooth vector fields on $M$ and $[\cdot, \cdot]$ represents the Lie bracket; namely, $[X, Y]=X \circ Y-Y \circ X$. The local representation of the curvature tensor satisfies

$$
R_{m j k l}=\left(\partial_{m} \Gamma_{j k}^{t}-\partial_{j} \Gamma_{m k}^{t}\right) g_{t l}+\left(\Gamma_{m t l} \Gamma_{j k}^{t}-\Gamma_{j t l} \Gamma_{m k}^{t}\right),
$$

where $\Gamma_{m j}^{k}=\Gamma_{m j t} g^{t k}$ and $\left(g^{t k}\right)$ is the inverse of the metric matrix $\left(g_{t k}\right)$.

The Ricci curvature and the sectional curvature are defined by

$$
\begin{gathered}
R_{m k}=R_{m j l k} g^{j l}, \\
K_{i j}=-\frac{R_{i j i j}}{g_{i i} g_{j j}-g_{i j}^{2}},
\end{gathered}
$$

respectively.

A curve $\xi(t)$ on $M$ is said to be a geodesic if its tangent vector $\dot{\xi}(t)$ is displaced parallel along the curve $\xi(t)$; that is,

$$
\nabla_{\dot{\xi}(t)} \dot{\xi}(t)=0
$$

and it has the following local form

$$
\frac{\mathrm{d}^{2} \theta^{k}}{\mathrm{~d} t^{2}}+\Gamma_{j m}^{k} \frac{\mathrm{d} \theta^{j}}{\mathrm{~d} t} \frac{\mathrm{d} \theta^{m}}{\mathrm{~d} t}=0 .
$$

Suppose that the solution of $(12)$ is $\theta(t ; \beta)$, where $\beta$ is a vector whose components are the integration constants.

Considering the wrapped Cauchy entropic dynamical model, the corresponding statistical manifold is

$$
S:=\left\{p(x ; \theta) \mid \theta=\left(\theta^{1}, \theta^{2}\right) \in \mathbb{R}_{+}^{2}\right\},
$$

where $p(x ; \theta)$ is given by (1) with parameters $\theta^{1}=\mu$ and $\theta^{2}=$ $\gamma$. The Fisher information matrix can be easily calculated as

$$
g=\operatorname{diag}\left(\frac{1}{2 \sinh ^{2} \theta^{2}}, \frac{1}{2 \sinh ^{2} \theta^{2}}\right) .
$$

Proposition 2. The nonzero components of the Ricci curvature are

$$
R_{11}=R_{22}=-\frac{1}{\sinh ^{2} \theta^{2}} .
$$

Proof. A direct calculation of (5) gives the nonvanishing Riemannian connection coefficients

$$
\Gamma_{112}=-\Gamma_{121}=-\Gamma_{211}=-\Gamma_{222}=\frac{\cosh \theta^{2}}{2 \sinh ^{3} \theta^{2}} .
$$

From the relation $\Gamma_{m j}^{k}=\Gamma_{m j t} g^{t k}$, one can get another type of connection coefficients

$$
\Gamma_{11}^{2}=-\Gamma_{12}^{1}=-\Gamma_{21}^{1}=-\Gamma_{22}^{2}=\frac{\cosh \theta^{2}}{\sinh \theta^{2}} .
$$

From (8), the following nonzero component of the curvature tensor is obtained:

$$
R_{1212}=\frac{1}{2 \sinh ^{4} \theta^{2}} .
$$

Therefore, the proof can be finished by a simple substitution of (18) into (9). 
The following theorem is obtained by substituting (18) and (14) into (10).

Theorem 3. S is a manifold with constant negative curvature $K=-2$.

\section{Topological and Geometric Properties of $S$}

Lemma 4. $S$ is diffeomorphic to $\mathbb{R}_{+}^{2}$ and hence simply connected.

Proof. The topology, as well as the smooth structure, of $S$ is induced by those on $\mathbb{R}_{+}^{2}$; hence $S$ is diffeomorphic to $\mathbb{R}_{+}^{2}$. The fact that $\mathbb{R}_{+}^{2}$ is simply connected implies that $S$ is also simply connected.

If $S$ were also complete, it would be a space form with constant negative curvature, which was isometric to the hyperbolic space $H^{2}(-2)$. However, $S$ is not complete. We begin with a necessary and sufficient condition of the completeness.

Definition 5 (see [19]). Let $(M, g)$ be a Riemannian manifold. A smooth curve $\gamma:[0,+\infty) \rightarrow M$ is called a divergent curve if for any compact subset $K \subset M$, there exists $t_{0}>0$, s.t., $\gamma\left(t_{0}\right) \notin K$. If $\gamma$ is a divergent curve, its length is defined by

$$
L(\gamma):=\lim _{s \rightarrow+\infty} \int_{0}^{s}\left|\gamma^{\prime}(t)\right| \mathrm{d} t .
$$

Lemma 6 (see [22]). ( $M, g, d)$ is a connected Riemannian manifold; then $M$ is complete if and only if every divergent curve has infinite length.

Proof. (1) Suppose that $M$ is complete and $\gamma$ is a divergent curve. Consider

$$
\bar{V}_{n}:=\{p \in M \mid d(p, \gamma(0)) \leq n\}, \quad \forall n \in \mathbb{N}
$$

where for each $n, \bar{V}_{n}$ is bounded and closed, hence compact. The divergence of $\gamma$ implies that, for all $n \in \mathbb{N}^{*}, \exists t_{n} \geq 0$, s.t., $\gamma\left(t_{n}\right) \notin \bar{V}_{n}$. As a result,

$$
\int_{0}^{t_{n}}\left|\gamma^{\prime}(t)\right| \mathrm{d} t \geq d\left(\gamma(0), \gamma\left(t_{n}\right)\right) \geq n ;
$$

hence $L(\gamma)=+\infty$.

(2) Suppose every divergent curve has infinite length. Assume that $M$ is noncomplete, which implies that $\exists p \in M$, $\exists v_{0} \in T_{p} M$, and $\gamma(t)=\exp _{p}\left(t v_{0}\right)$ is only defined on $t \in[0,1)$.

$L(\gamma)<\left|v_{0}\right|<+\infty$ means $\gamma$ is not divergent; that is, there exists a compact subset $K \subset M$ s.t. $\gamma(t) \in K$, for all $t$. Choose sequence $\left\{t_{n}\right\}_{n=1}^{+\infty}$, s.t., $t_{n} \rightarrow 1$ and $t_{n}<t_{n+1}$, for all $n \in \mathbb{N}$. It is obvious that $d\left(\gamma\left(t_{n}\right), \gamma\left(t_{m}\right)\right) \rightarrow 0$ when $n, m$ are sufficiently large. Hence $\left\{\gamma\left(t_{n}\right)\right\}$ is a Cauchy sequence. The completeness of $M$ implies that there exists $q \in K$ s.t. $\gamma\left(t_{n}\right) \rightarrow q$.

Then $\gamma(1):=q$ is a extension of $\gamma$ from $[0,1)$ to $[0,1]$ which contradicts to the assumption, which implies that $M$ is in fact complete.
Theorem 7. S is noncomplete.

Proof. Suppose that $\gamma(t):=(0, t) \in \mathbb{R}_{+}^{2}, t \in[1,+\infty)$ is a curve along the $y$-axis. It is obvious that $\gamma$ is divergent. However, the length of $\gamma$ satisfies

$$
\begin{aligned}
L(\gamma) & =\int_{1}^{+\infty}\left|\gamma^{\prime}(t)\right| \mathrm{d} t \\
& =\int_{1}^{+\infty} \frac{1}{\sqrt{2} \sinh (t)} \mathrm{d} t \\
& =\left.\frac{1}{\sqrt{2}} \ln \left(\tanh \left(\frac{t}{2}\right)\right)\right|_{1} ^{+\infty} \\
& =\frac{\ln (e+1)-\ln (e-1)}{\sqrt{2}}<+\infty .
\end{aligned}
$$

This implies $S$ is noncomplete by Lemma 6 .

Although $S$ is neither isometric to the hyperbolic space nor a symmetric space, it is "partly symmetric," which is presented in Lemma 10.

Definition 8. Let $I(S)$ be the group of isometric transformations on $S$. A family of transformations on $S$ are defined as

$$
\varphi_{\alpha}: S \longrightarrow S, \quad\left(\theta^{1}, \theta^{2}\right) \longmapsto\left(2 \alpha-\theta^{1}, \theta^{2}\right), \quad \forall \alpha \in \mathbb{R} .
$$

Remark 9. $\varphi_{\alpha}$ is just the reflection about the line $\theta^{1}=\alpha$, which is a line parallel to the $y$-axis.

Lemma 10. $\varphi_{\alpha} \in I(S)$, for all $\alpha \in \mathbb{R}$.

Proof. For any fixed $\alpha \in \mathbb{R}$, for all $p=\left(\theta^{1}, \theta^{2}\right) \in S, q:=$ $\varphi_{\alpha}(p)=\left(2 \alpha-\theta^{1}, \theta^{2}\right)$.

Consider

$$
\begin{gathered}
\gamma_{1}(t):=\left(\theta^{1}+t, \theta^{2}\right), \quad \gamma_{2}(t):=\left(\theta^{1}, \theta^{2}+t\right), \\
\left(\varphi_{\alpha}\right)_{* p}\left(\frac{\partial}{\partial \theta^{1}}\right)=\left.\frac{\mathrm{d}}{\mathrm{d} t}\right|_{t=0}\left(\varphi_{\alpha} \circ \gamma_{1}(t)\right) \\
=\left.\frac{\mathrm{d}}{\mathrm{d} t}\right|_{t=0}\left(2 \alpha-\theta^{1}-t, \theta^{2}\right)=-\left.\frac{\partial}{\partial \theta^{1}}\right|_{q}, \\
\left(\varphi_{\alpha}\right)_{* p}\left(\frac{\partial}{\partial \theta^{2}}\right)=\left.\frac{\mathrm{d}}{\mathrm{d} t}\right|_{t=0}\left(\varphi_{\alpha} \circ \gamma_{2}(t)\right) \\
g_{q}\left(\left(\varphi_{\alpha}\right)_{* p}\left(\frac{\partial}{\partial \theta^{1}}\right),\left(\varphi_{\alpha}\right)_{t=0}\left(\frac{\partial}{\partial \theta^{1}}\right)\right) \\
=g_{q}\left(-\frac{\partial}{\partial \theta^{1}},-\frac{\partial}{\partial \theta^{1}}\right)_{t}=\frac{1}{2 \sinh ^{2} \theta^{2}}=g_{p}\left(\frac{\partial}{\partial \theta^{1}}, \frac{\partial}{\partial \theta^{1}}\right),
\end{gathered}
$$




$$
\begin{gathered}
g_{q}\left(\left(\varphi_{\alpha}\right)_{* p}\left(\frac{\partial}{\partial \theta^{1}}\right),\left(\varphi_{\alpha}\right)_{* p}\left(\frac{\partial}{\partial \theta^{2}}\right)\right) \\
=g_{q}\left(-\frac{\partial}{\partial \theta^{1}}, \frac{\partial}{\partial \theta^{2}}\right)=0=g_{p}\left(\frac{\partial}{\partial \theta^{1}}, \frac{\partial}{\partial \theta^{2}}\right), \\
g_{q}\left(\left(\varphi_{\alpha}\right)_{* p}\left(\frac{\partial}{\partial \theta^{2}}\right),\left(\varphi_{\alpha}\right)_{* p}\left(\frac{\partial}{\partial \theta^{2}}\right)\right) \\
=g_{q}\left(\frac{\partial}{\partial \theta^{2}}, \frac{\partial}{\partial \theta^{2}}\right)=\frac{1}{2 \sinh ^{2} \theta^{2}}=g_{p}\left(\frac{\partial}{\partial \theta^{2}}, \frac{\partial}{\partial \theta^{2}}\right) .
\end{gathered}
$$

Hence $\varphi_{\alpha}$ is an isometry.

As a result, $G:=\left\{\varphi_{\alpha} \mid \alpha \in \mathbb{R}\right\}$ is a subgroup of $I(S)$. Lemma 10 implies that $S$ is "almost" homogeneous, although not truly homogeneous. In fact, $S$ is a cohomogeneity one manifold, defined in Definition 11.

Definition 11. Let $(M, g)$ be a Riemannian manifold and $I(M)$ is its group of isometric transformations. Then $I(M)$ has a natural action $\Phi$ on $M$ :

$$
\begin{array}{r}
\Phi: I(M) \times M \longrightarrow M, \quad(\varphi, p) \longmapsto \varphi(p), \\
\forall \varphi \in I(M), \quad p \in M .
\end{array}
$$

$M$ is a cohomogeneity one manifold if the codimension of orbit space of this action is 1 .

Remark 12. The codimension of the orbit space is closely related to the homogeneous properties. For example, if $M$ is a homogeneous space, then the orbit space is whole manifold $M$, and hence its codimension is 0 . The less that codimension is, the more homogeneous the manifold is. In other words, a cohomogeneity one manifold is "the most homogeneous manifold" except for the real ones.

Actually, cohomogeneity one manifolds are natural generalizations of homogeneous manifolds. The systematic study of cohomogeneity one manifolds was started by Bergery, who successfully constructed new invariant Einstein metrics on cohomogeneity one manifolds. In addition, Bryant and Salamon constructed special metrics with exceptional holonomy groups $G_{2}$ and $\operatorname{Spin}(7)$ on these manifolds [23-30].

We state a well known result without proof before Theorem 14.

Lemma 13. Every homogeneous space is complete.

Theorem 14. S is a cohomogeneity one manifold.

Proof. Let $p=\left(\theta^{1}, \theta^{2}\right) \in S$ and $I(S)_{p}:=\{\varphi(p) \mid \varphi \in I(S)\}$ be its orbit space. If $\operatorname{dim}\left(I(S)_{p}\right)=2, S$ will be homogeneous. Lemma 13 implies that $S$ is complete, which contradicts to Theorem 7. This contradiction implies $\operatorname{dim}\left(I(S)_{p}\right) \leq 1$.

On the other hand,

$$
\begin{aligned}
G_{p} & :=\{\varphi(p) \mid \varphi \in G\}=\left\{\varphi_{\alpha}(p) \mid \alpha \in \mathbb{R}\right\} \\
& =\left\{\left(2 \alpha-\theta^{1}, \theta^{2}\right) \mid \alpha \in \mathbb{R}\right\}=\left\{\left(x, \theta^{2}\right) \mid x \in \mathbb{R}\right\} .
\end{aligned}
$$

Since $G$ is a subgroup of $I(S), G_{p} \subset I(S)_{p}$. This implies that

$$
\operatorname{dim}\left(I(S)_{p}\right) \geq \operatorname{dim}\left(G_{p}\right)=1 .
$$

Based on the two inequalities, we conclude that $\operatorname{dim}\left(I(S)_{p}\right)=$ 1 ; hence its codimension is 1 , which means that $S$ is a cohomogeneity one manifold.

\section{Instability Analysis}

In this section, we will calculate some geodesics and investigate the instability of the geodesics by the Jacobi vector fields.

Combining (12) and (17), the geodesic equations of the WCED model are given by the following differential system:

$$
\begin{gathered}
\frac{\mathrm{d}^{2} \theta^{1}}{\mathrm{~d} t^{2}}-\frac{2 \cosh \theta^{2}}{\sinh \theta^{2}} \frac{\mathrm{d} \theta^{1}}{\mathrm{~d} t} \frac{\mathrm{d} \theta^{2}}{\mathrm{~d} t}=0, \\
\frac{\mathrm{d}^{2} \theta^{2}}{\mathrm{~d} t^{2}}+\frac{\cosh \theta^{2}}{\sinh \theta^{2}}\left(\frac{\mathrm{d} \theta^{1}}{\mathrm{~d} t}\right)^{2}-\frac{\cosh \theta^{2}}{\sinh \theta^{2}}\left(\frac{\mathrm{d} \theta^{2}}{\mathrm{~d} t}\right)^{2}=0 .
\end{gathered}
$$

Generally, these equations are quite hard to be solved; however we can get some special geodesics based on the cohomogeneity one property.

Lemma 15. All lines parallel to the y-axis are geodesics.

Proof. It is well known that the fixed-points set of any isometry is a geodesic. Since $\varphi_{\alpha}$ is an isometry and its fixedpoints set is the line $x=\theta^{1}$, the lemma follows.

According to Lemma 15, the equation of such geodesic satisfies

$$
\theta^{1}=\theta_{0}^{1}
$$

Hence (28) can be reduced to

$$
\begin{gathered}
\frac{\mathrm{d} \theta^{1}}{\mathrm{~d} t}=0 \\
\frac{\mathrm{d}^{2} \theta^{2}}{\mathrm{~d} t^{2}}-\frac{\cosh \theta^{2}}{\sinh \theta^{2}}\left(\frac{\mathrm{d} \theta^{2}}{\mathrm{~d} t}\right)^{2}=0
\end{gathered}
$$

Then solution is expressed as

$$
\begin{gathered}
\theta^{1}=a_{3}, \\
\theta^{2}=\ln \left|\frac{a_{2} \exp \left\{a_{1} t\right\}+1}{a_{2} \exp \left\{a_{1} t\right\}-1}\right| .
\end{gathered}
$$

Consider the parameter family of geodesics

$$
\mathscr{F}(C)=\left\{\theta^{l}(t, C)\right\}^{l=1,2},
$$

where $\theta^{l}$ is a geodesic satisfying (28) and $C$ is an integration constant vector. The length of geodesics in $\mathscr{F}(C)$ is defined as

$$
\Theta(t ; \beta):=\int\left(g_{i j} \mathrm{~d} \theta^{i} \mathrm{~d} \theta^{j}\right)^{1 / 2} \text {. }
$$


Therefore, the length of geodesics on WCED model is obtained as

$$
\Theta(t ; C)=\int_{0}^{t} \sqrt{g_{11}\left(\frac{\mathrm{d} \theta^{1}}{\mathrm{~d} t}\right)^{2}+g_{22}\left(\frac{\mathrm{d} \theta^{2}}{\mathrm{~d} t}\right)^{2}} \mathrm{~d} \tau=\frac{\sqrt{2 C_{1}}}{2} t,
$$

where $C_{1}$ is constant. In order to investigate the behavior of two neighboring geodesics labeled by the parameter $C_{1}$, we consider the following difference:

$$
\Delta \Theta=\left(\frac{\sqrt{2\left(C_{1}+\delta C_{1}\right)}}{2}-\frac{\sqrt{2 C_{1}}}{2}\right) t .
$$

It is clear that $\Delta \Theta$ diverges; that is, the length of two neighboring geodesics with slightly different parameters $C_{1}$ and $C_{1}+\delta C_{1}$ differs in a remarkable way as $t \rightarrow \infty$.

The stability of the geodesics is completely determined by the curvature of manifold. Studying the stability of dynamics means determining the evolution of perturbations of geodesics. For isotropic manifolds, the geodesic spread is unstable only if their constant sectional curvatures are negative. As long as the curvatures are negative, the geodesic spread is unstable even if the manifold is no longer isotropic [19]. However, it is more attractive to know the divergent degree of a geodesic.

The Jacobi vector field $J$, that is, the evolution perturbation vector, satisfies the following (Jacobi) equation:

$$
\nabla_{\dot{\xi}(t)} \nabla_{\dot{\xi}(t)} J=R(\dot{\xi}(t), J) \dot{\xi}(t)
$$

where $\xi(t)$ is a geodesic on manifold $M$. It is also called the geodesic derivation equation, as it has close connections with the geodesic. In general, the Jacobi equations are difficult to solve, but in particular this manifold has a constant negative curvature, which is of great help. Given a normal geodesic $\xi$ and supposing $J$ is a normal Jacobi vector field along $\xi(t)$, then

$$
\begin{aligned}
R(\dot{\xi}(t), J) \dot{\xi}(t) & =2(g(\dot{\xi}(t), \dot{\xi}(t)) J-g(\dot{\xi}(t), J) \dot{\xi}(t)) \\
& =2 J
\end{aligned}
$$

Thus the Jacobi equation is reduced to

$$
\frac{\mathrm{d}^{2} J(t)}{\mathrm{d} t^{2}}-2 J(t)=0
$$

Choose a unit orthogonal frame $\left\{e_{i}(t)\right\}$, s.t., $e_{2}(t)=\dot{\xi}(t)$ and suppose

$$
J(t)=J^{i}(t) e_{i}(t)
$$

Then the Jacobi equation satisfies

$$
\begin{gathered}
\frac{\mathrm{d}^{2} J^{1}(t)}{\mathrm{d} t^{2}}-2 J^{1}(t)=0, \\
J^{2}(t)=0 .
\end{gathered}
$$

The solutions are

$$
\begin{gathered}
J^{1}(t)=C_{1} e^{\sqrt{2} t}+C_{2} e^{-\sqrt{2} t}, \\
J^{2}(t)=0 .
\end{gathered}
$$

This means that the geodesic spread on manifold is described by means of an exponential order divergent Jacobi vector field.

\section{Conclusion and Remarks}

In this paper, we investigate the manifold of wrapped Cauchy distributions. By considering the geometric structures of the WCED, we conclude that the WCED is a constant negative curvature space with $K=-2$. By a series of lemmas and theorems, we prove that it is a cohomogeneity one manifold. This example is interesting because of its statistical background, while some other examples are deliberately constructed. In addition, we calculate the geodesics and the Jacobi vector field by some tricks based on some special properties of $S$. As a result, the divergent behavior of geodesics can be described as an exponential order divergent Jacobi vector field.

\section{Conflict of Interests}

The authors declare that there is no conflict of interests regarding the publication of this paper.

\section{Authors' Contribution}

The authors declare that the study was realized in collaboration with the same responsibility. All authors read and approved the final paper.

\section{Acknowledgments}

This work is supported by the National Natural Science Foundations of China (nos. 11126161 and NSFC61440058), Beijing Higher Education Young Elite Teacher Project (no. YETP 0388), and the Fundamental Research Funds for the Central Universities (no. FRF-BR-12-005).

\section{References}

[1] S. Amari, Differential Geometrical Methods in Statistics, Springer, Berlin, Germany, 1990.

[2] S. Amari and H. Nagaoka, Methods of Information Geo metry, Oxford University Press, Oxford, UK, 2000.

[3] T. Li, L. Peng, and H. Sun, "The geometric structure of the inverse gamma distribution," Beiträge zur Algebra und Geometrie, vol. 49, no. 1, pp. 217-225, 2008.

[4] L. Peng, H. Sun, D. Sun, and J. Yi, "The geometric structures and instability of entropic dynamical models," Advances in Mathematics, vol. 227, no. 1, pp. 459-471, 2011.

[5] L. Peng, H. Sun, and G. Xu, "Information geometric characterization of the complexity of fractional Brownian motions," Journal of Mathematical Physics, vol. 53, no. 12, Article ID 123305, 12 pages, 2012. 
[6] L. Peng, H. Sun, and X. Sun, "Geometric structure of Hamiltonian dynamics with conformal Eisenhart metric," International Journal of Mathematics and Mathematical Sciences, vol. 2011, Article ID 710274, 26 pages, 2011.

[7] A. Caticha, "Entropic dynamics," in Bayesian Inference and Maximum Entropy Methods in Science and Engineering, R. L. Fry, Ed., AIP Conference Proceedings, pp. 617-302, 2002.

[8] C. Cafaro and S. A. Ali, "Jacobi fields on statistical manifolds of negative curvature," Physica D: Nonlinear Phenomena, vol. 234, no. 1, pp. 70-80, 2007.

[9] K. V. Mardia and P. E. Jupp, Directional Statistics, John Wiley \& Sons, New York, NY, USA, 1999.

[10] C. Bingham, "An antipodally symmetric distribution on the sphere," The Annals of Statistics, vol. 2, pp. 1201-1225, 1974.

[11] J. T. Kent, "The Fisher-Bingham distribution on the sphere," Journal of the Royal Statistical Society, vol. 44, pp. 71-80, 1982.

[12] R. A. Fisher, "Dispersion on a sphere," Proceedings of the Royal Society of London A, vol. 217, pp. 295-305, 1953.

[13] N. I. Fisher, Statistical Analysis of Circular Data, Cambridge University Press, Cambridge, UK, 1996.

[14] G. Borradaile, Statistics of Earth Science Data, Springer, New York, NY, USA, 2003.

[15] K. A. Keating and S. Cherry, "Modeling utilization distributions in space and time," Ecology, vol. 90, no. 7, pp. 1971-1980, 2009.

[16] H.-I. Wu, B.-L. Li, T. A. Springer, and W. H. Neill, "Modelling animal movement as a persistent random walk in two dimensions: expected magnitude of net displacement," Ecological Modelling, vol. 132, no. 1-2, pp. 115-124, 2000.

[17] Y. Vermard, E. Rivot, S. Mahévas, P. Marchal, and D. Gascuel, "Identifying fishing trip behaviour and estimating fishing effort from VMS data using Bayesian Hidden Markov Models," Ecological Modelling, vol. 221, no. 15, pp. 1757-1769, 2010.

[18] K. Thorup, J. Rabøl, and J. J. Madsen, "Can clock-and-compass explain the distribution of ringing recoveries of pied flycatchers?" Animal Behaviour, vol. 60, no. 2, pp. F3-F8, 2000.

[19] M. P. do Carmo, Riemannian Geometry, Birkhäuser, Boston, Mass, USA, 1992.

[20] P. Petersen, Riemannian Geometry, Springer, New York, NY, USA, 2nd edition, 2006.

[21] C. R. Rao, "Information and the accuracy attainable in the estimation of statistical parameters," Bulletin of the Calcutta Mathematical Society, vol. 37, pp. 81-91, 1945.

[22] W. Chen and X. Li, Introduction to Riemannian Geometry, Peking University Press, Beijing, China, 2012.

[23] D. Alekseevsky, "Riemannian manifolds of cohomogeneity one," in Proceedings of the Colloquium on Differential Geometry, vol. 56 of Colloeq. Math. Soc. J. Bolyai, pp. 9-22, 1989.

[24] A. Alekseevsky and D. Alekseevsky, "G-manifold with onedimensional orbit space," Advances in Soviet Mathematics, vol. 8, pp. 1-31, 1992.

[25] A. Besse, Einstein Manifolds, Springer, Berlin, Germany, 1987.

[26] R. L. Bryant, "Metrics with holonomy $G_{2}$ or spin (7)," in Arbeitstagung Bonn 1984, vol. 1111 of Lecture Notes in Mathematics, pp. 269-277, Springer, Berlin, Germany, 1985.

[27] R. L. Bryant, "A survey of Riemannian metrics with special holonomy groups," in Proceedings of the International Congress of Mathematicians, vol. 1, pp. 505-514, AMS, Berkeley, Calif, USA, 1987.

[28] R. L. Bryant, "Metrics with exceptional holonomy," Annals of Mathematics, Second Series, vol. 126, no. 3, pp. 525-576, 1987.
[29] R. Bryant and F. Harvery, "Some remarks on the geometry of manifolds with exceptional holonomy," preprint, 1994.

[30] R. L. Bryant and S. M. Salamon, "On the construction of some complete metrics with exceptional holonomy," Duke Mathematical Journal, vol. 58, no. 3, pp. 829-850, 1989. 


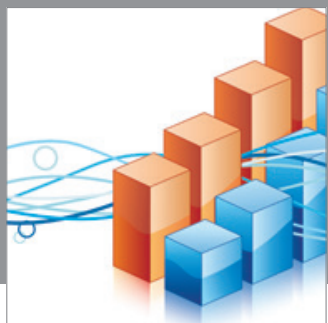

Advances in

Operations Research

mansans

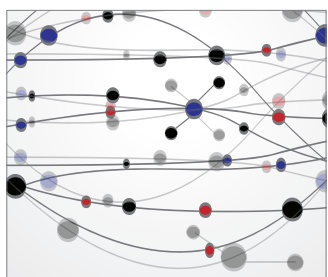

The Scientific World Journal
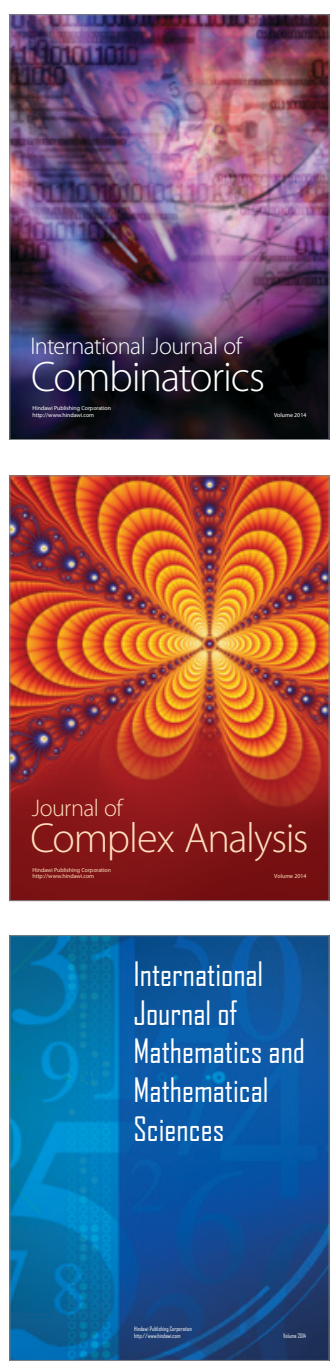
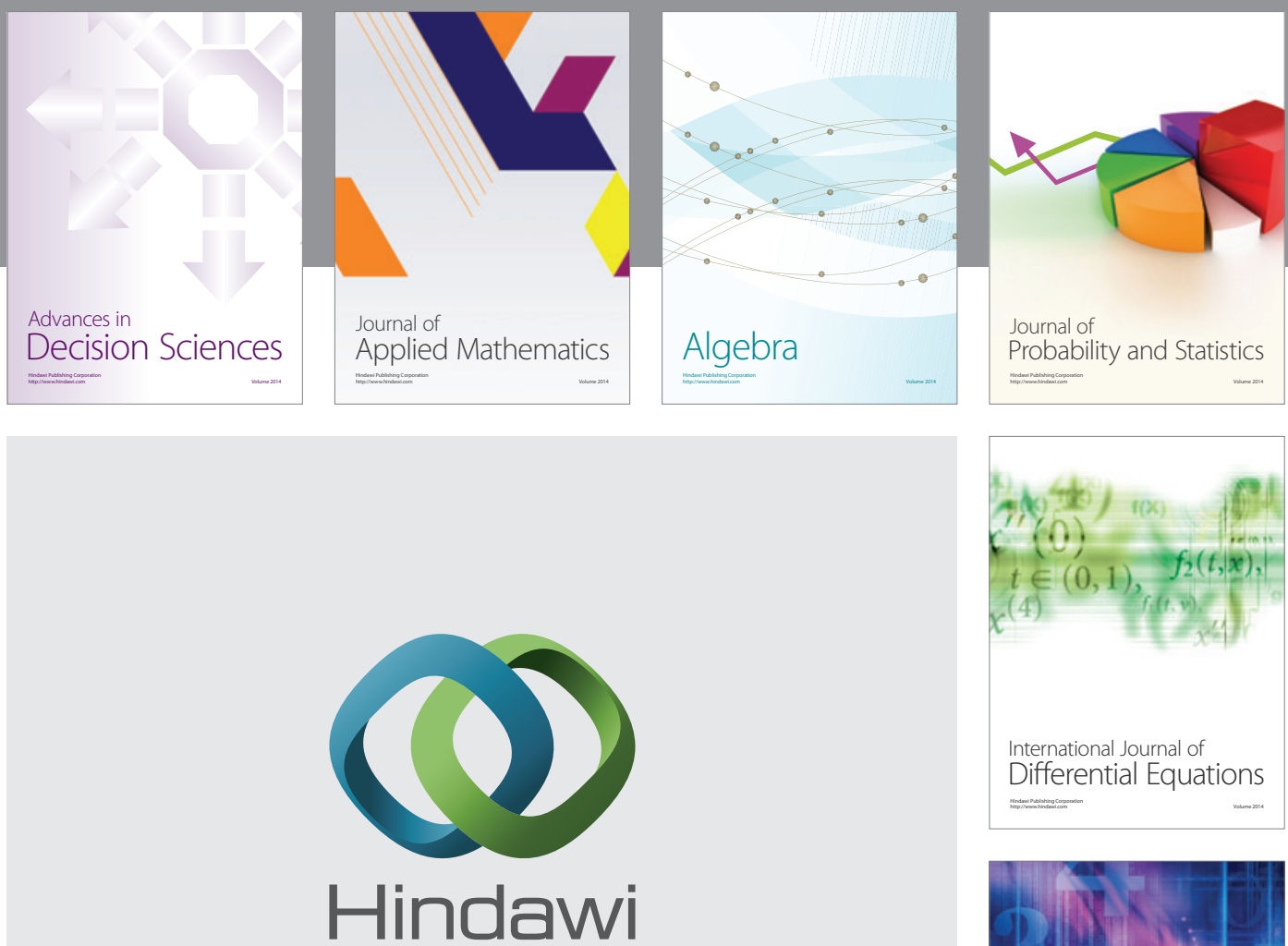

Submit your manuscripts at http://www.hindawi.com
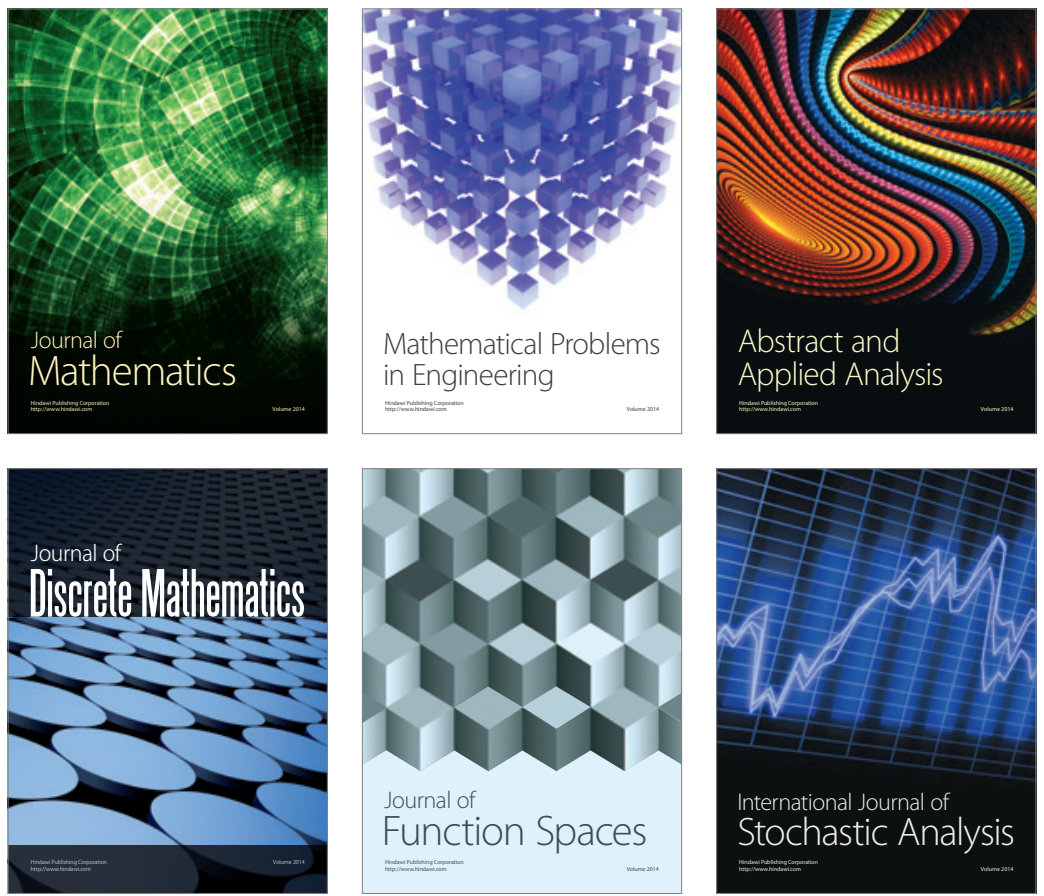

Journal of

Function Spaces

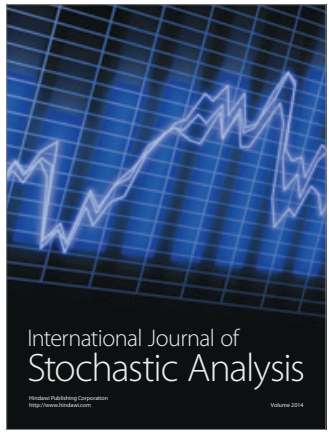

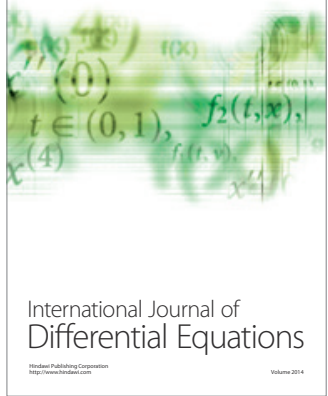
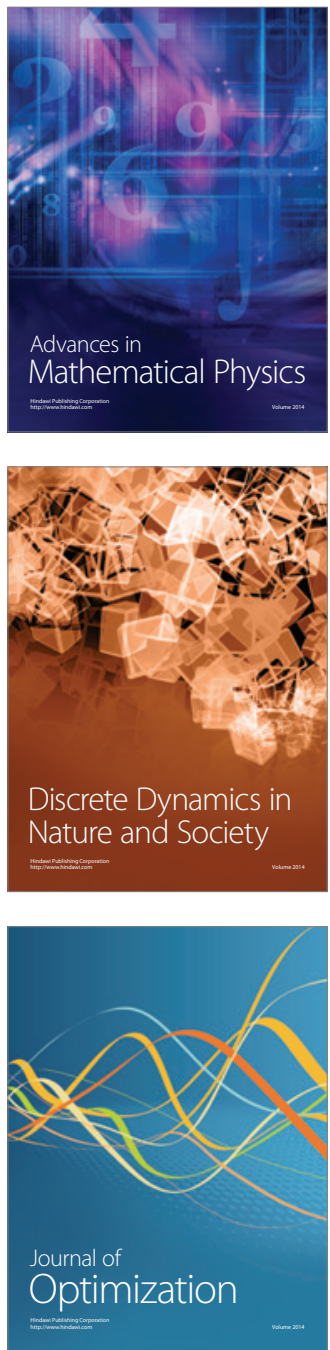